\title{
Trans-vaginal specimen extraction following totally laparoscopic subtotal gastrectomy in early gastric cancer
}

\author{
Sang-Ho Jeong $\cdot$ Young-Joon Lee $\cdot$ Won Jun Choi $\cdot$ Won Young Paik $\cdot$ \\ Chi-Young Jeong $\cdot$ Soon-Tae Park $\cdot$ Sang-Kyung Choi $\cdot$ Soon-Chan Hong $\cdot$ \\ Eun-Jung Jung $\cdot$ Young-tae Joo $\cdot$ Woo-Song Ha
}

Received: 31 July 2010/ Accepted: 21 September 2010/Published online: 25 January 2011

(C) The International Gastric Cancer Association and The Japanese Gastric Cancer Association 2011

\begin{abstract}
Background Although natural orifice extraction is now widely performed, there have been no reports of this procedure following subtotal gastrectomy for gastric cancer. This report describes trans-vaginal specimen extraction in four patients with early gastric cancer.

Methods The clinical data of four patients with early gastric cancer were reviewed. Totally laparoscopic subtotal gastrectomy and $\mathrm{D} 1+\beta$ lymph node dissection was performed using five trocars and a conventional procedure. Posterior colpotomy was performed by an experienced gynecologist, who retrieved the specimens in a retrieval bag via the trans-vaginal route. The colpotomy site was repaired immediately following specimen removal. Reconstruction was performed using the intracorporeal Billroth II method and an endo-GIA 60.
\end{abstract}

S.-H. Jeong · Y.-J. Lee $(\bowtie) \cdot$ C.-Y. Jeong - S.-T. Park ·

S.-K. Choi - S.-C. Hong · E.-J. Jung - Y. Joo - W.-S. Ha

Department of Surgery, Gyeongsang National University

Hospital, 90 Chilam-dong, Jinju, Gyeongsang South Province

660-702, South Korea

e-mail: yjleegnu@gmail.com

W. J. Choi · W. Y. Paik

Department of Obstetrics and Gynecology, Gyeongsang National University Hospital, Jinju, South Korea

S.-H. Jeong · Y.-J. Lee · W. J. Choi · W. Y. Paik ·

C.-Y. Jeong · S.-T. Park · S.-K. Choi - S.-C. Hong ·

E.-J. Jung - Y. Joo · W.-S. Ha

Gyeongnam Regional Cancer Center, Jinju, South Korea

S.-H. Jeong · Y.-J. Lee · W. J. Choi · W. Y. Paik ·

C.-Y. Jeong $\cdot$ S.-T. Park $\cdot$ S.-K. Choi $\cdot$ S.-C. Hong ·

E.-J. Jung - Y. Joo - W.-S. Ha

Gyeongsang Institute of Health Sciences, Jinju, South Korea
Results Totally laparoscopic subtotal gastrectomy and trans-vaginal specimen extraction was successfully accomplished in all patients without intraoperative complications.

Conclusions The present technique may be a safe and feasible operative procedure for some limited groups of elderly female patients with early gastric cancer.

Keywords Stomach neoplasms - Laparoscopy · Trans-vaginal $\cdot$ Natural orifice $\cdot$ Natural orifice specimen extraction

\section{Introduction}

Despite a decrease in its incidence and associated mortality in Korea, gastric cancer remains the second most frequent cause of death in Korea [1]. Nearly a million new cases are diagnosed each year in the world, and it is the fourth most common type of cancer and the second leading cause of death worldwide [2,3].

The advantages of laparoscopic surgery over open surgery in terms of quality of life include reduced pain, earlier recovery, a shorter hospital stay, and a better cosmetic outcome [4-10]. Surgical approaches have shifted from multiport surgery, extracorporeal anastomosis, and minilaparotomy incision to reduced-port or single-port surgery, intracorporeal anastomosis, and natural orifice extraction. However, there have been no reports in the international literature of trans-vaginal extraction in early gastric cancer. This report describes trans-vaginal specimen extraction in four female patients with early gastric cancer.

\section{Patients, materials, and methods}

The clinical data of four patients with early gastric cancer were reviewed. All four patients underwent trans-vaginal 
specimen extraction following totally laparoscopic subtotal gastrectomy at Gyeongsang National University Hospital between July 2009 and May 2010.

\section{Surgical procedure}

\section{Positioning and placement of trocars}

Following the administration of a general anesthetic, the patient was placed in the lithotomy position. The abdomen, pubis, and vagina were draped. The surgeon stood to the right of the patient and the first assistant stood opposite the surgeon. The camera operator stood between the legs of the patient. The umbilical camera port was established using an open technique. After establishing a pneumoperitoneum, five ports were placed (two 11- $\mathrm{mm}$, one 12-mm, and two 5-mm ports; Fig. 1). Intracorporeal $\mathrm{CO}_{2}$ pressure was maintained at $12 \mathrm{mmHg}$. The operation commenced with a survey of the intraabdominal cavity. The cavity was inspected to exclude signs of: (1) any tumor seeding in the peritoneum or mesentery; (2) distant metastasis; or (3) direct invasion of the tumor into adjacent organs.

\section{Localization of the tumor}

Intraoperative gastrofibroscopy was performed to determine the location of the tumor and the resection margin. The tumor site was marked using gentian violet solution under endoscopic guidance.

\section{Laparoscopic subtotal gastrectomy}

The surgical procedure involved: (1) totally laparoscopic subtotal gastrectomy; (2) partial omentectomy; and (3) $\mathrm{D} 1+\beta$ lymph node dissection $(1,3,4 \mathrm{~d}, 4 \mathrm{sb}, 5,6,7,8,9$, $11 \mathrm{p})$. The main procedure began with the mobilization of the greater curvature of the stomach, using ultrasonically activated coagulating shears (LCS) (SonoSurg; Olympus, Aomori, Japan) and LigaSure (Valleylab, Boulder, CO, USA). Partial omentectomy was performed in all four patients because early gastric cancer was suspected. The dissection proceeded towards the lower pole of the spleen. The left gastroepiploic vessels were ligated. The dissection then proceeded towards the right gastroepiploic vessels. The right gastroepiploic artery was divided at its root after clipping, and the infrapyloric lymph nodes were dissected. The posterior aspect of the duodenal stump was mobilized to allow visualization of the gastroduodenal artery. An incision was made in the lesser omentum between the first portion of the duodenum and the right gastric artery. The duodenum was then transected with an endoscopic linear stapler under laparoscopic observation.
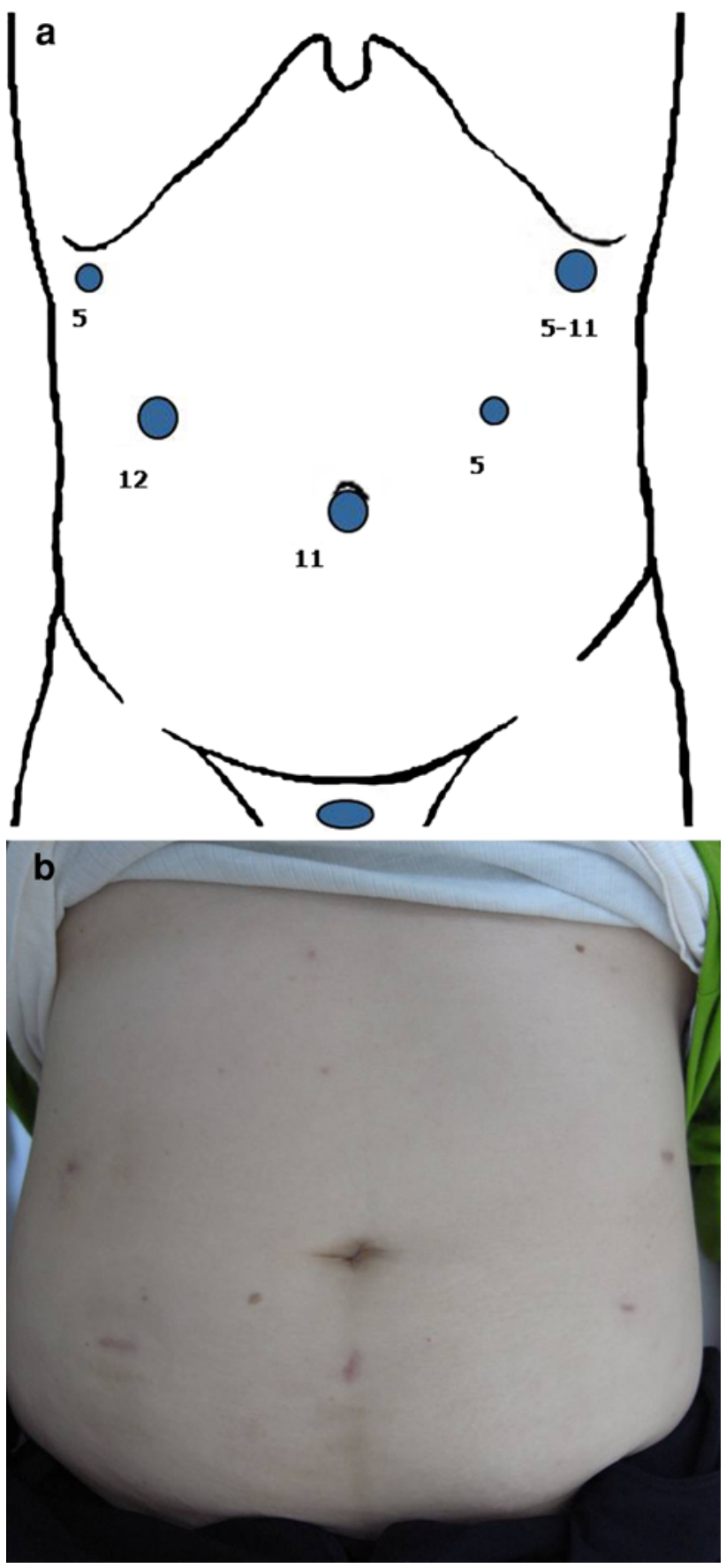

Fig. 1 a Placement of trocars. b Trocar sites 3 months postoperatively

The lymph nodes of the common hepatic artery were dissected, and the right gastric artery was divided after clipping. The lesser omentum was divided and the left gastric vessel was clipped at its root. The lymph nodes along the celiac axis and the left gastric artery were then dissected. The perigastric lymph nodes along the lesser curvature were dissected up to the site of the esophagogastric junction. The proximal stomach was divided using 
two endo-GIA 60 (Covidien, Mansfield, MA, USA). A retrieval bag (Lap Bag; Sejong, Paju, Korea) was introduced through the transabdominal trocar, and the specimen was transferred into it. The retrieval bag was then transferred into the pelvic cavity for trans-vaginal extraction.

\section{Trans-vaginal specimen extraction}

Prior to the surgery, the patients had undergone bimanual pelvic examination and a Papanicolaou (Pap) test at the outpatient clinic of the Department of Obstetrics and Gynecology. In all four patients, clinical examination showed no evidence of vaginal narrowing or obliteration of the pouch of Douglas, and the Pap test was negative. After gastric resection, a posterior colpotomy was performed by an experienced gynecologist. A weighted speculum was positioned to retract the posterior vaginal wall and Deaver retractors were used to further expose the uterine cervix. A tenaculum was placed on the anterior lip of the cervix. Following exposure of the cervix, the uterine cavity was measured with a probe. The cervix was dilated progressively to a diameter of $6 \mathrm{~mm}$. The uterus was manipulated using a Kronner Manipujector (CooperSurgical, Trumbull, CT, USA).

The posterior lip of the cervix was grasped with a tenaculum and the cervix was elevated anteriorly. The area of the posterior fornix in which the posterior vaginal wall meets the cervix was identified. The colpotomy site was located approximately $1-2 \mathrm{~cm}$ posterior to this junction. Two Allis clamps were used to elevate the area and a $2-\mathrm{cm}$ transverse cut was made using curved Mayo scissors. Access to the space of Douglas was achieved using a blunt, extra-long 10-mm trocar $(150 \mathrm{~mm}$; Johnson and Johnson, Ethicon Endosurgery Inc, Cincinnati, OH, USA) under laparoscopic observation.

The retrieval bag was gently withdrawn until it reached the intraabdominal tip of the vaginal port. The retrieval bag containing the specimen was removed together with the trans-vaginal trocar. The incision in the posterior fornix was closed with an intravaginal 2-0 Vicryl (Ethicon A division of J \& J Medical Limited, Livingston, UK) suture (Fig. 2).

\section{Reconstruction}

Reconstruction was performed $30 \mathrm{~cm}$ distal to the Treiz ligament by using an endo-GIA 60 (ENDO GIA universal straight 60 3.5; Covidien) according to the intracorporeal Billroth II method (antecolic, isoperistaltic type gastrojejunostomy). The endo-GIA insertion site was repaired by the insertion of intracorporeal interrupted sutures or an intracorporeal continuous suture, using an Endostitch (Covidien; Fig. 3).
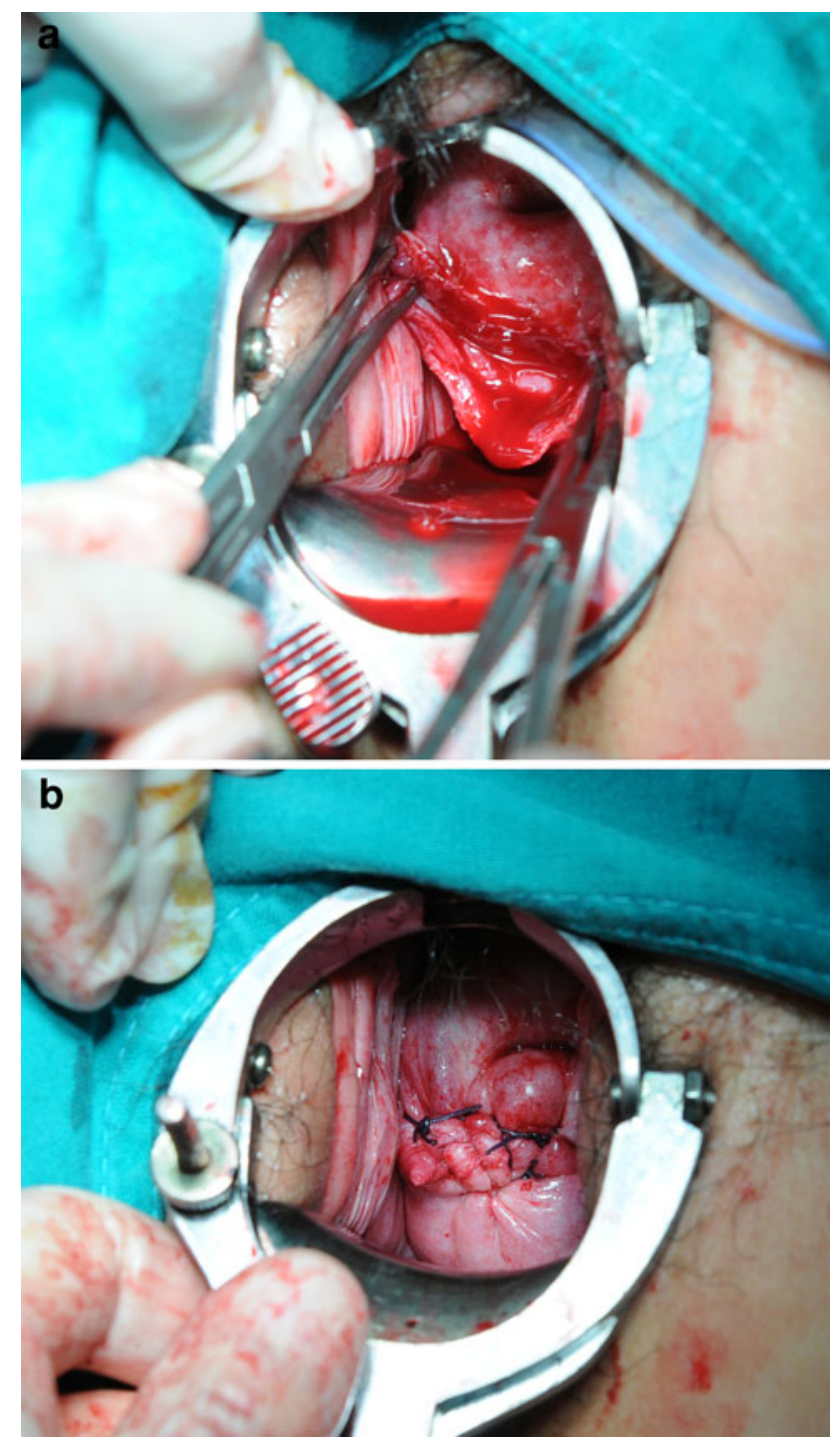

Fig. 2 Posterior colpotomy; a following removal of the gastric specimen; b post-repair

\section{Postoperative course}

Three days postoperatively, each patient was allowed to take sips of water, even in the absence of any postoperative passage of gas. A soft fluid diet was introduced 4-5 days postoperatively, and the patient was allowed to eat soft bread 6 days postoperatively. Three of the patients were discharged 7-8 days postoperatively.

We experienced one case (patient 1; Table 1) of postoperative bleeding in one patient, who subsequently followed a different postoperative course. In this patient, the hemoglobin level decreased 2 days post-surgery and a computed tomography (CT) scan revealed the presence of a nonbleeding intraabdominal hematoma around the site of resection. The patient recovered with supportive care only (absolute bed rest and transfusion). She was able to take 


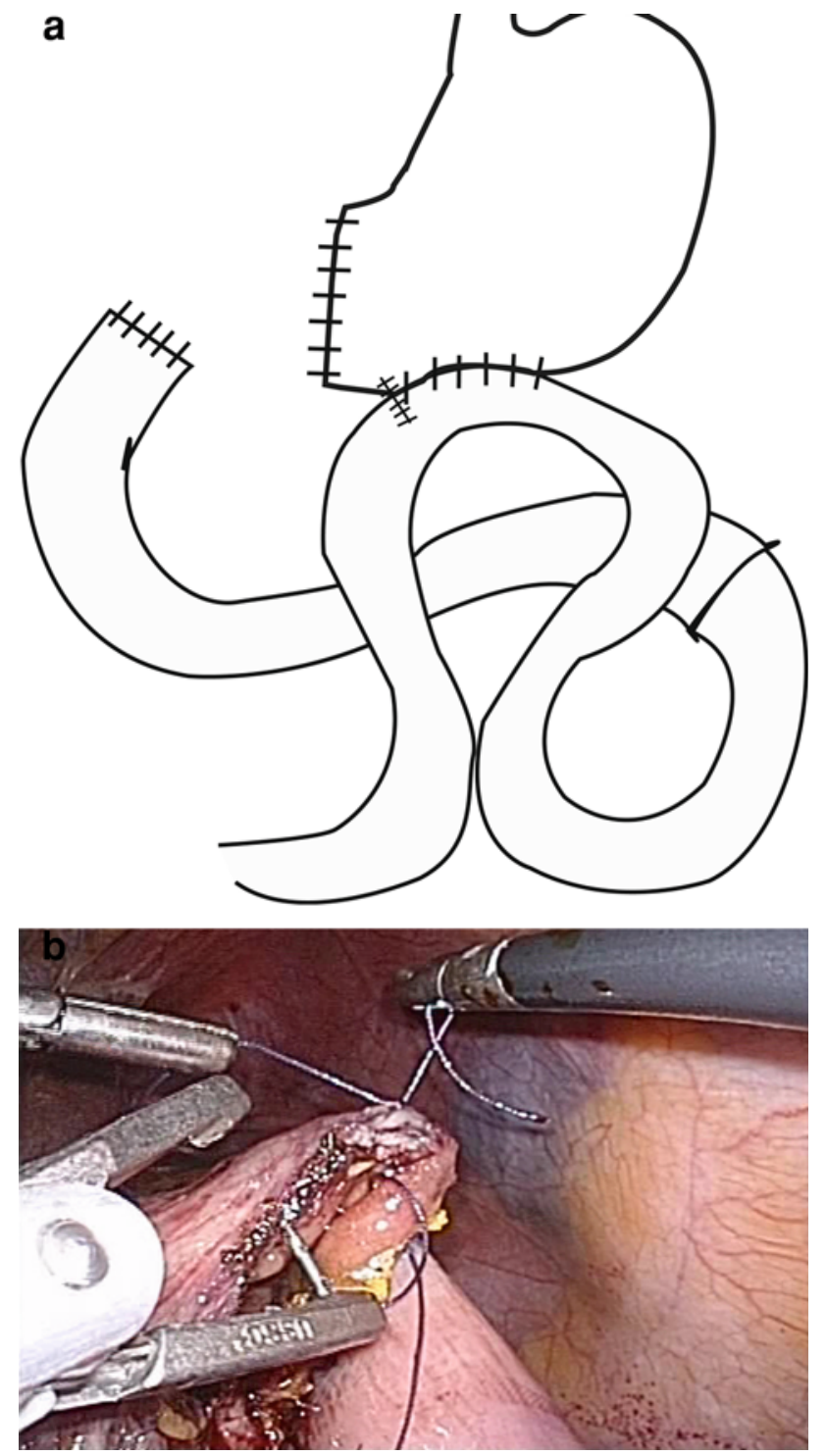

Fig. 3 a Schematic diagram of the reconstruction. b Laparoscopic repair of endo-GIA insertion site using Endostitch (Covidien)

sips of water 5 days postoperatively, and was discharged 5 days later.

\section{Results}

Four patients participated in the study. The mean age of the patients was 67.5 years, and the mean body mass index (BMI) was 25.1. Patient one experienced epigastric discomfort before the gastro-endoscopy, but the other patients had no symptoms; however, early gastric cancer was detected using gastro-endoscopic screening. One patient had hypertension, one had diabetes mellitus, and a third patient had both comorbid disorders. Two patients had an asymptomatic gallbladder (GB) stone. The tumor was located on the greater curvature aspect of the antrum in three patients. In the fourth patient, the tumor was located on the lower anterior wall of the body of the stomach. In two patients, cholecystectomy was also performed during the procedure for asymptomatic gallstones. The mean operating time was $358 \mathrm{~min}$. No intraoperative complications occurred in any of the four patients. We experienced postoperative bleeding in patient 1 , but this patient recovered with only supportive care. Histological examination confirmed the presence of mucosal invasion in patients 1 , 2 , and 4 and submucosal invasion in patient 3 . The mean number of retrieved lymph nodes was 16.3 . In patient 2 , a metastatic lymph node was found. The mean postoperative hospital stay was 8 days (Table 1).

\section{Discussion}

The first reported trans-vaginal extraction was performed in 1993 by Delvaux et al. [11]. They described the extraction of the gallbladder and a large gallbladder stone $(>4 \mathrm{~cm})$ via the trans-vaginal route following laparoscopic cholecystectomy in female patients. In 1996, Kim et al. [12] described trans-vaginal extraction of the rectum in four patients following low anterior resection, with excellent results. Gill et al. [13] reported vaginal extraction of the intact specimen following laparoscopic radical nephrectomy in 2002. Recently, Palanivelu et al. [14] reported extraction of total colon and rectum following totally laparoscopic proctocolectomy. However, there have been no previous reports of trans-vaginal extraction following gastrectomy. Trans-vaginal endoscopic partial gastrectomy was reported in a porcine model in 2008 [15]. This technique was developed as an extension of natural orifice transluminal endoscopic surgery (NOTES). Nakajima et al. [16] subsequently reported partial gastrectomy using NOTES in patients with gastric submucosal tumors.

There are four potential natural orifice specimen extraction (NOSE) routes: transgastric, transrectal, transvaginal, and transurethral. Given the large volume of gastric specimens, use of the transgastric and transurethral route for stomach specimen extraction is not feasible. Transrectal extraction is feasible, but carries a high risk of infection and postoperative leakage. In contrast, there have been no reports of any increased risk of postoperative leakage or infection for the trans-vaginal route [17], and the use of this route was therefore selected for the present study. However, the use of this route is limited to women who have had sexual intercourse. Previous studies have excluded young nulliparous females, and patients with atrophic vaginitis, vaginal infection, vaginal prolapse syndrome, or severe pelvic adhesions [13]. In two studies that compared abdominal and vaginal route surgery, no 
Table 1 Patient characteristics

\begin{tabular}{|c|c|c|c|c|}
\hline & Case 1 & Case 2 & Case 3 & Case 4 \\
\hline Age (years) & 58 & 69 & 67 & 76 \\
\hline Presenting symptoms & Epigastric discomfort & None & None & None \\
\hline BMI & 27.7 & 26.1 & 26.1 & 25.1 \\
\hline Co-morbid disease & $\mathrm{DM}$ & GB stone & HT & HT, DM, GB stone \\
\hline Gross type & IIc & $\mathrm{IIb}+\mathrm{IIc}$ & III & IIa \\
\hline Tumor size $(\mathrm{cm})$ & $1 \times 0.9$ & $4.5 \times 2.4$ & $2 \times 2$ & $2.3 \times 1.6$ \\
\hline Tumor depth & Mucosal & Mucosal & Submucosal & Mucosal \\
\hline Tumor location & Lower body, anterior wall & Antrum, greater curvature & Antrum, greater curvature & Antrum, greater curvature \\
\hline Number of retrieved LNs & 12 & 23 & 17 & 13 \\
\hline Metastatic LN & 0 & 1 & 0 & 0 \\
\hline WHO classification & MD & MD & MD & WD \\
\hline Lauren classification & Intestinal & Intestinal & Intestinal & Intestinal \\
\hline TNM stage & Ia & $\mathrm{Ib}$ & Ia & Ia \\
\hline Operating time $(\min )$ & 370 & 315 & 355 & 395 \\
\hline Combined organ resection & No & GB & No & GB \\
\hline Duration of hospital stay (days) & 10 & 7 & 7 & 8 \\
\hline
\end{tabular}

$B M I$ body mass index, $L N$ lymph node, $D M$ diabetes mellitus, $G B$ gallbladder, $H T$ hypertension, $M D$ moderately differentiated, $W D$ well differentiated

postoperative differences in vaginal sensation, ability to achieve orgasm, pregnancy rate, or rate of dyspareunia were observed [18, 19]. In the present study, patient 2 had a history of hysterectomy. However, safe use of the trans-vaginal route was achieved following laparoscopic adhesiolysis.

The introduction of intracorporeal anastomosis in laparoscopic gastrectomy was one of the factors that led to the development of single-port surgery or NOSE, because intracorporeal anastomosis does not necessitate a minilaparotomy. Intracorporeal Billroth II was first reported by Goh et al. [20] in patients with peptic ulceration. In 1998, Kuo et al. [21] reported the first case of totally laparoscopic BII gastrectomy with lymph node dissection. The greatest technical difficulty in this type of laparoscopic surgery is the establishment of an intracorporeal anastomosis. However, this difficulty can be overcome by the use of various procedures, including a delta-shaped anastomosis, totally laparoscopic uncut Roux-en-Y, and totally laparoscopic Roux-en-Y using a linear stapler [22, 23]. Ikeda et al. [24] reported that the performance of total laparoscopic distal gastrectomy reduced the time required for minilaparotomy, as well as reducing the time to the first ingestion of oral fluids and food, reducing the duration of hospital stay, and reducing the serum C-reactive protein (CRP) level.

Single-port surgery and NOTES are now widely performed and have been adapted for use in a range of procedures. There have been numerous reports of sleeve gastrectomy performed via single-port surgery [25, 26]. However, the use of single-port surgery in gastric cancer remains limited to the dissection of lymph nodes. In the future, we intend to develop a robotic instrument not only for single-port surgery, but also for the NOTES procedure.

In conclusion, trans-vaginal specimen extraction following totally laparoscopic subtotal gastrectomy may be a safe and feasible procedure for some limited groups of elderly female patients with early gastric cancer. The present study assessed a small patient sample, and studies of larger patient samples are required to confirm these findings.

\section{References}

1. Lee HJ, Yang HK, Ahn YO. Gastric cancer in Korea. Gastric Cancer. 2002;5:177-82.

2. Kamangar F, Dores GM, Anderson WF. Patterns of cancer incidence, mortality, and prevalence across five continents: defining priorities to reduce cancer disparities in different geographic regions of the world. J Clin Oncol. 2006;24:2137-50.

3. Yang HK, Association IcotKGC. 2004 Nationwide gastric cancer report in Korea. J Korean Gastric Cancer Assoc. 2007;7:47-54.

4. Adachi Y, Suematsu T, Shiraishi N, Katsuta T, Morimoto A, Kitano S, et al. Quality of life after laparoscopy-assisted Billroth I gastrectomy. Ann Surg. 1999;229:49-54.

5. Horiuchi T, Shimomatsuya T, Chiba Y. Laparoscopically assisted pylorus-preserving gastrectomy. Surg Endosc. 2001;15:325-8.

6. Huscher CG, Anastasi A, Crafa F, Recher A, Lirici MM. Laparoscopic gastric resections. Semin Laparosc Surg. 2000;7:26-54.

7. Kim YW, Baik YH, Yun YH, Nam BH, Kim DH, Choi IJ, et al. Improved quality of life outcomes after laparoscopy-assisted distal gastrectomy for early gastric cancer: results of a prospective randomized clinical trial. Ann Surg. 2008;248:721-7. 
8. Mochiki E, Kamimura H, Haga N, Asao T, Kuwano H. The technique of laparoscopically assisted total gastrectomy with jejunal interposition for early gastric cancer. Surg Endosc. 2002;16:540-4.

9. Uyama I, Sugioka A, Fujita J, Komori Y, Matsui H, Hasumi A. Laparoscopic total gastrectomy with distal pancreatosplenectomy and D2 lymphadenectomy for advanced gastric cancer. Gastric Cancer. 1999;2:230-4.

10. Uyama I, Sugioka A, Matsui H, Fujita J, Komori Y, Hatakawa Y, et al. Laparoscopic side-to-side esophagogastrostomy using a linear stapler after proximal gastrectomy. Gastric Cancer. 2001;4:98-102.

11. Delvaux G, Devroey P, De Waele B, Willems G. Trans-vaginal removal of gallbladders with large stones after laparoscopic cholecystectomy. Surg Laparosc Endosc. 1993;3:307-9.

12. Kim J, Shim M, Kwun K. Laparoscopic-assisted trans-vaginal resection of the rectum. Dis Colon Rectum. 1996;39:582-3.

13. Gill IS, Cherullo EE, Meraney AM, Borsuk F, Murphy DP, Falcone T. Vaginal extraction of the intact specimen following laparoscopic radical nephrectomy. J Urol. 2002;167:238-41.

14. Palanivelu C, Rangarajan M, Jategaonkar PA, Anand NV. An innovative technique for colorectal specimen retrieval: a new era of "natural orifice specimen extraction" (N.O.S.E). Dis Colon Rectum. 2008;51:1120-4.

15. Nakajima K, Takahashi T, Souma Y, Shinzaki S, Yamada T, Yoshio T, et al. Trans-vaginal endoscopic partial gastrectomy in porcine models: the role of an extra endoscope for gastric control. Surg Endosc. 2008;22:2733-6.

16. Nakajima K, Nishida T, Takahashi T, Souma Y, Hara J, Yamada $\mathrm{T}$, et al. Partial gastrectomy using natural orifice translumenal endoscopic surgery (NOTES) for gastric submucosal tumors: early experience in humans. Surg Endosc. 2009;23:2650-5.

17. Swain P. Nephrectomy and natural orifice translumenal endoscopy (NOTES): trans-vaginal, transgastric, transrectal, and transvesical approaches. J Endourol. 2008;22:811-8.
18. Roovers JP, van der Bom JG, van der Vaart $\mathrm{CH}$, van Leeuwen JH, Scholten PC, Heintz AP. A randomized comparison of postoperative pain, quality of life, and physical performance during the first 6 weeks after abdominal or vaginal surgical correction of descensus uteri. Neurourol Urodyn. 2005;24:334-40.

19. Paraiso MF, Barber MD, Muir TW, Walters MD. Rectocele repair: a randomized trial of three surgical techniques including graft augmentation. Am J Obstet Gynecol. 2006;195:1762-71.

20. Goh P, Tekant Y, Kum CK, Isaac J, Shang NS. Totally intraabdominal laparoscopic Billroth II gastrectomy. Surg Endosc. 1992;6:160.

21. Kuo WH, Lee WJ, Chen CN, Yuan RH, Yu SC. Laparoscopic subtotal gastrectomy with lymphadenectomy in a patient with early gastric cancer. J Formos Med Assoc. 1998;97:127-30.

22. Kanaya S, Gomi T, Momoi H, Tamaki N, Isobe H, Katayama T, et al. Delta-shaped anastomosis in totally laparoscopic Billroth I gastrectomy: new technique of intraabdominal gastroduodenostomy. J Am Coll Surg. 2002;195:284-7.

23. Takaori K, Nomura E, Mabuchi H, Lee SW, Agui T, Miyamoto $\mathrm{Y}$, et al. A secure technique of intracorporeal Roux-Y reconstruction after laparoscopic distal gastrectomy. Am J Surg. 2005;189:178-83.

24. Ikeda O, Sakaguchi Y, Aoki Y, Harimoto N, Taomoto J, Masuda $\mathrm{T}$, et al. Advantages of totally laparoscopic distal gastrectomy over laparoscopically assisted distal gastrectomy for gastric cancer. Surg Endosc. 2009;23:2374-9.

25. Saber AA, El-Ghazaly TH. Early experience with SILS port laparoscopic sleeve gastrectomy. Surg Laparosc Endosc Percutan Tech. 2009;19:428-30.

26. Varela JE. Single-site laparoscopic sleeve gastrectomy: preclinical use of a novel multi-access port device. Surg Innov. 2009;16:207-10. 